\title{
Intraoperative Frozen Section May Reduce the Need for Reoperative Thyroid Surgery in Patients with Follicular Neoplasm
}

\author{
Dena Arumugam ${ }^{\mathrm{a}, \mathrm{d}}$, Carlos A. Macias ${ }^{\mathrm{a}}$, Renee L. Arlow ${ }^{\mathrm{a}}$, Sumana Narayanan ${ }^{\mathrm{a}}$, Shou-En Lu ${ }^{\mathrm{b}}$, \\ Malik Deen ${ }^{\mathrm{c}}$, Michael Mayc, Renee L. Artymyshync ${ }^{\mathrm{c}}$, Nicola J. Barnard ${ }^{\mathrm{c}}$, Tomer Davidov ${ }^{\mathrm{a}}$, \\ Stanley Z. Trooskin ${ }^{\mathrm{a}}$
}

\begin{abstract}
Background: Follicular neoplasm (Bethesda IV cytology) requires thyroid lobectomy for diagnosis. Approximately $15-30 \%$ of cases are ultimately malignant, requiring reoperative completion thyroidectomy. We sought to reassess whether intraoperative frozen section (FS) reduces the need for reoperative thyroidectomy in these cases.

Methods: A retrospective chart review of fine needle aspiration (FNA) results showing follicular neoplasm from 2003 to 2012 was performed. Our practice is to offer thyroid lobectomy with FS for these patients; with suspicious FS, surgery proceeded to total thyroidectomy. FS suspicious for papillary thyroid carcinoma (PTC) was based on nuclear features (nuclear grooves/atypia, pseudo-nuclear inclusions) and for follicular thyroid carcinoma (FTC) was based on capsular/vascular invasion.

Results: A total of 148 patients with follicular neoplasm on FNA were identified, of which 79 underwent FS. Fifteen had suspicious FS with 12 having malignancy on final pathology, for a positive predictive value of $80 \%$. Nine showed features suspicious for PTC, of which eight had PTC. Six showed features suspicious for FTC, of which four had malignancy on final pathology. Of the 64 patients with unremarkable FS, 49 had benign final pathology, for a negative predictive value of $77 \%$. Twelve (19\%) patients required reoperation. Seven of 19 patients (37\%) with malignancy avoided reoperation due to FS. Risk of malignancy with follicular neoplasm diagnosis was $34 \%(51 / 148)$, while risk of malignancy with follicular neoplasm and suspicious FS was $80 \%$.
\end{abstract}

Manuscript accepted for publication October 20, 2015

aRutgers Robert Wood Johnson Medical School, Department of Surgery, Clinical Academic Building CAB 7300, 125 Paterson Street, New Brunswick, NJ 08901, USA

${ }^{b}$ Rutgers School of Public Health, Department of Biostatistics, 683 Hoes Lane West, Piscataway, NJ 08854, USA

'Rutgers Robert Wood Johnson Medical School, Department of Pathology, 1 Robert Wood Johnson Place, MEB 212, New Brunswick, NJ 08903, USA

${ }^{\mathrm{d} C}$ Corresponding Author: Dena Arumugam, Rutgers Robert Wood Johnson Medical School, 125 Paterson Street, MEB 596, New Brunswick, NJ 08901, USA. Email: dena.arumugam@gmail.com

doi: http://dx.doi.org/10.14740/jcs286w
Conclusions: Risk of malignancy with follicular neoplasm and suspicious FS is $80 \%$. FS may avoid reoperative completion thyroidectomy in up to a third of patients.

Keywords: Follicular neoplasm; Frozen section; Thyroidectomy; Reoperation; Papillary thyroid cancer; Follicular thyroid cancer

\section{Introduction}

Workup of a solitary thyroid nodule requires a multimodal approach, including a detailed history and physical examination, blood testing, imaging and biopsy. Fine needle aspiration (FNA), which is typically performed via ultrasound guidance, is the gold standard for biopsy of thyroid nodules. The Bethesda system for classifying thyroid nodules was created to provide a comprehensive, uniform way of risk stratifying and managing solitary thyroid nodules based on FNA results [1]. This study focuses on patients with Bethesda IV cytology, that of follicular neoplasm, a more difficult pathologic diagnosis to manage. Follicular neoplasm carries a $15-30 \%$ risk of malignancy [2,3], and therefore often requires an operation for diagnosis [4]. Therefore, for patients undergoing thyroid lobectomy, there is $15-30 \%$ probability of having papillary thyroid carcinoma (PTC) or follicular carcinoma that will require reoperative completion thyroidectomy [2].

Intraoperative frozen section (FS) is used by some to guide surgical management of patients with a cytological diagnosis of follicular neoplasm. Its use, however, is controversial. Many believe that FS of indeterminate thyroid nodules may be limited in detecting thyroid malignancies especially in follicular or Hurthle cell lesions [5, 6], because it produces discordant diagnoses with final pathology due to sampling error $[7,8]$, distorts sample architecture $[8,9]$, and is time consuming $[10$, $11]$ and costly $[10,11]$ without improved patient outcomes. Others suggest that the routine use of FS in thyroid surgery supplements information from FNA biopsy and leads to better surgical decision making [12-16].

It is our goal in this study to reassess the use of intraoperative FS in those patients with FNA biopsy showing follicular neoplasm and determine whether FS results could reduce the need for reoperative completion thyroidectomy. 
Table 1. Demographic Information for 79 Patients With a Preoperative Fine Needle Aspiration Diagnosis of Follicular Neoplasm/Lesion Who Underwent Intraoperative Frozen Section

\begin{tabular}{|lll|}
\hline & $\begin{array}{l}\text { Number of } \\
\text { patients }\end{array}$ & $\begin{array}{l}\text { Percent of } \\
\text { patients }\end{array}$ \\
\hline Total patients with frozen section & 79 & \\
\hline Female & 57 & $72 \%$ \\
Family history of thyroid disease & 24 & $30 \%$ \\
History of radiation exposure & 9 & $11 \%$ \\
\hline History of tobacco use & 27 & $34 \%$ \\
\hline
\end{tabular}

\section{Methods}

This was a retrospective chart review performed at Rutgers Robert Wood Johnson Medical School of all patients with a solitary thyroid nodule that was diagnosed by preoperative FNA as follicular neoplasm or follicular lesion (Bethesda IV cytology). Cases from 2003 until 2012 were analyzed.

All patients were treated by the same surgeons (T.D. and S.Z.T.) with the same practice pattern; that is, thyroid lobectomy with intraoperative FS was offered to all patients with an FNA diagnosis of follicular neoplasm. If FS was reported to be benign by intraoperative pathologic examination, thyroid lobectomy alone was performed. If FS exhibited features suspicious for cancer, total thyroidectomy was performed instead. Indeterminate FS reports were treated with thyroid lobectomy alone and ultimate management was deferred to final pathologic analysis.

FS was reported as suspicious for PTC when nuclear atypia, nuclear grooves, or pseudo-nuclear inclusions were present. Capsular or vascular invasion on FS was a sign of possible follicular thyroid carcinoma (FTC).

A total of three pathologists reviewed the FNA and FS specimens. For this chart review, results of the preoperative FNA and intraoperative FS analyses were obtained from the cytology and histopathology reports.

\section{Results}

A total of 148 patients had a preoperative FNA diagnosis of follicular neoplasm during the study time period. Of these, 79 underwent intraoperative FS. Sixty-nine patients underwent total thyroidectomy as an alternative to thyroid lobectomy and FS was not performed. Reasons for total thyroidectomy at initial operation included patient preference, patient risk factors (radiation exposure or family history of thyroid cancer), or characteristics of the thyroid nodules (increasing nodule size or contralateral nodules $>1.0 \mathrm{~cm}$ ).

\section{Patient demographics}

Mean patient age was 51 years (range 16 - 79 years) at the time of surgery. Fifty-seven patients were female and 22 were male (ratio of 2.6:1). Twenty-four patients had a family history of thyroid disease. Nine patients reported a prior history of radiation exposure. Twenty-seven patients had an extensive history of tobacco use (Table 1).

\section{Nodule characteristics}

Ultrasonography was the primary diagnostic tool and was used in $96 \%$ of patients. Most $(92 \%)$ patients had nodules on pre-

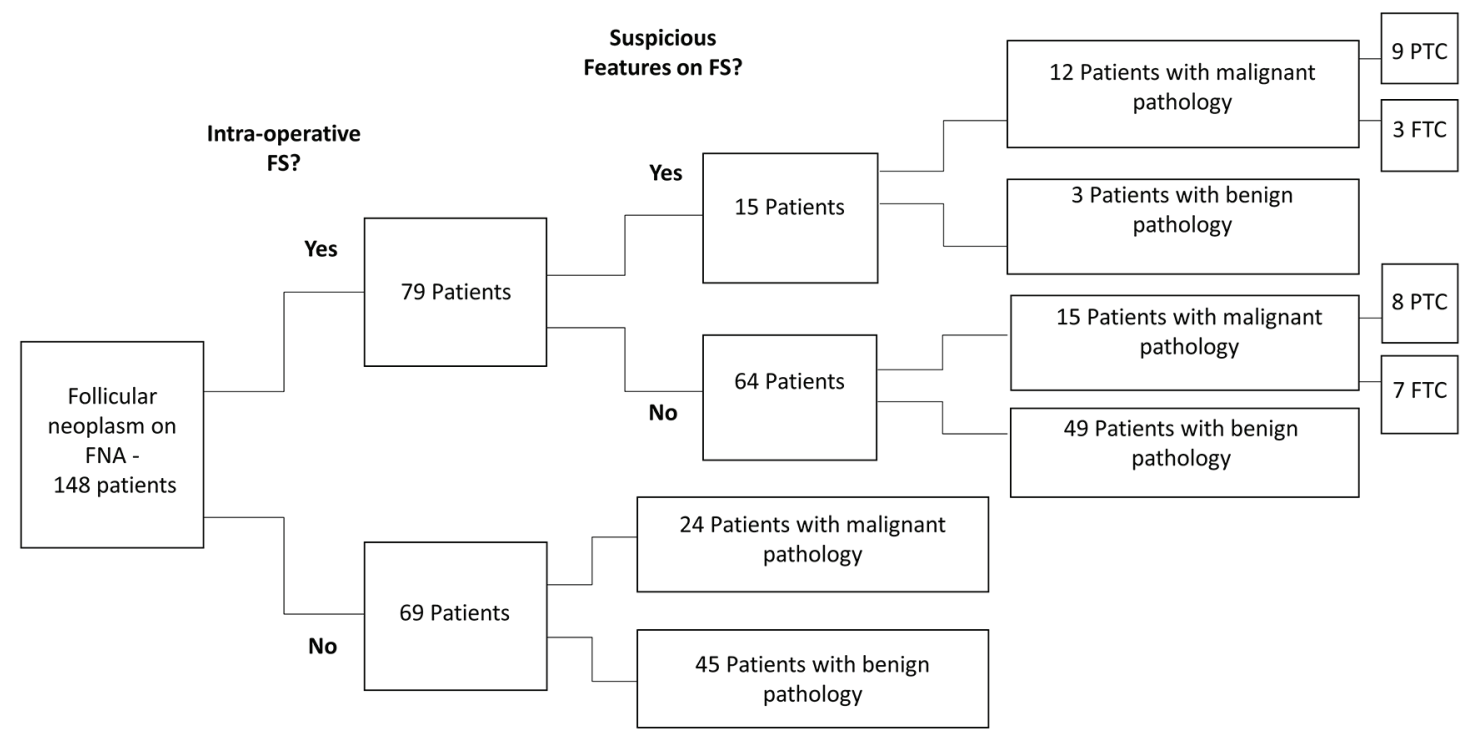

Figure 1. Patients with a preoperative fine needle aspiration (FNA) diagnosis of follicular neoplasm or follicular lesion and their intraoperative frozen section (FS) diagnosis and final pathology. PTC: papillary thyroid carcinoma. FTC: follicular thyroid carcinoma. 


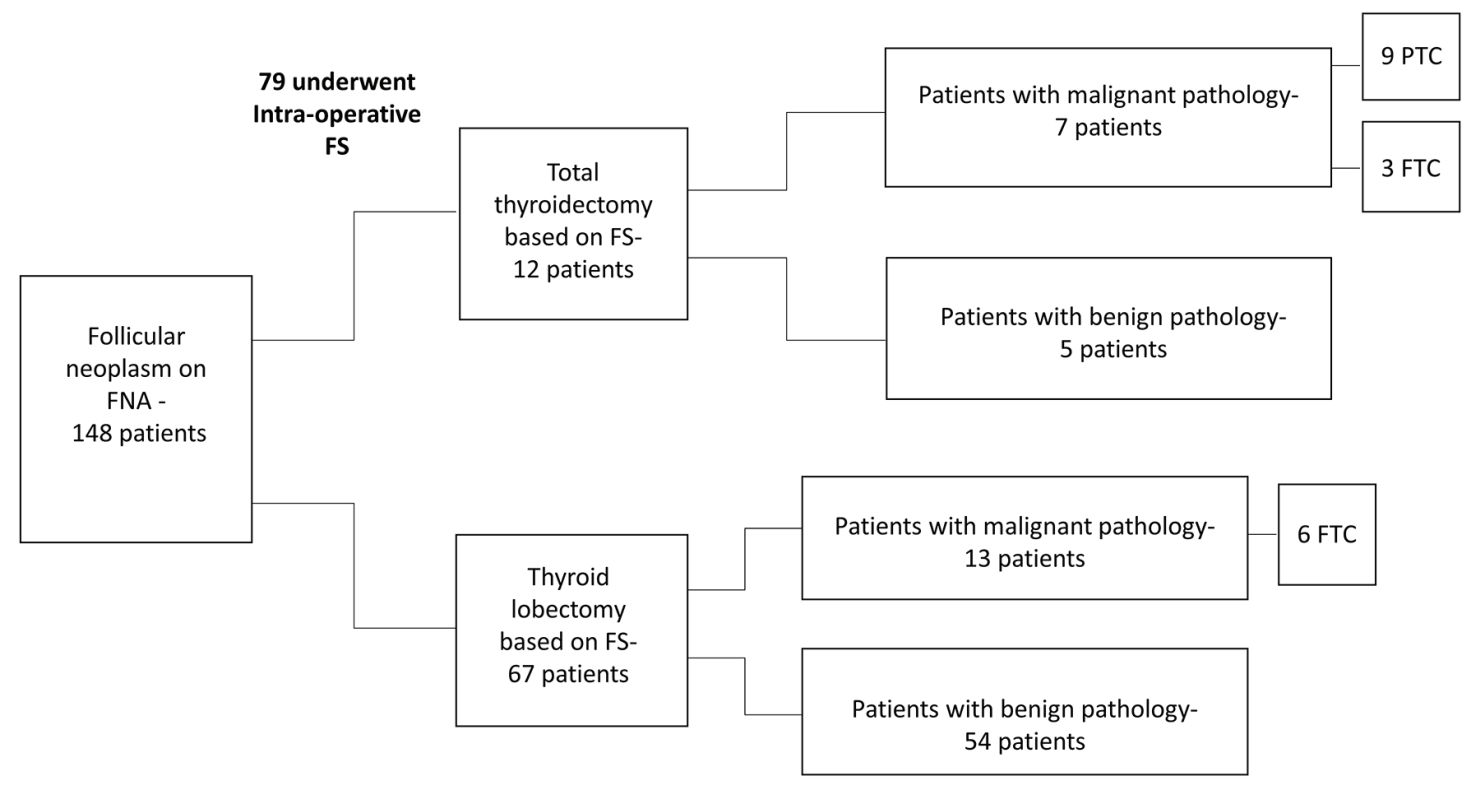

Figure 2. Patients with a preoperative fine needle aspiration (FNA) diagnosis of follicular neoplasm or follicular lesion and their operative management based on frozen section (FS) diagnosis and final pathology. PTC: papillary thyroid carcinoma. FTC: follicular thyroid carcinoma.

operative imaging that measured $>1.0 \mathrm{~cm}$. Forty-five $(57 \%)$ cases had nodules which were larger than $2.0 \mathrm{~cm}$ in size and $10(13 \%)$ nodules measured greater than $4.0 \mathrm{~cm}$ in size. Thirtyeight $(48 \%)$ patients showed the presence of contralateral nodules on imaging. Six patients $(8 \%)$ had calcifications within the dominant nodule, of which four (67\%) cases showed carcinoma on final pathologic examination.

\section{FS and final pathology}

Of the 79 patients with FNA diagnosis of follicular neoplasm who underwent intraoperative FS analysis, 15 had features suspicious of carcinoma on FS. Twelve $(80 \%)$ of these cases had a final pathologic diagnosis of malignancy, nine were positive for PTC, and three were positive for FTC.

Sixty-four patients had features on FS which were not suggestive of cancer. Of these 64 cases, $15(23 \%)$ had a diagnosis of malignancy on final pathology. Eight cases showed PTC and the remaining seven were positive for FTC.

These findings suggest a positive predictive value of $80 \%$ and a negative predictive value of $77 \%$ for intraoperative FS in FNA diagnosis of follicular neoplasm (Fig. 1).

Of the 69 patients with FNA diagnosis of follicular neoplasm who did not undergo intraoperative FS analysis, 24 had a malignant diagnosis on final pathology.

\section{FS and operative management}

Of the 79 patients who underwent intraoperative FS, 12 had findings on FS that warranted total thyroidectomy. Seven $(58 \%)$ of these patients had malignancy on final pathology, for which total thyroidectomy was appropriate management. Four patients had PTC and three had FTC. The remaining 67 patients proceeded with thyroid lobectomy alone based on FS findings. Thirteen (19\%) of these patients had malignancy on final pathology that required reoperative total thyroidectomy. Six cases had PTC and the remaining seven were positive for FTC.

In sum, 19 of the 79 patients warranted total thyroidectomy based on final pathologic diagnosis; seven (37\%) of these 19 patients underwent total thyroidectomy at their initial operation based on suspicious findings on FS (Fig. 2).

\section{Discussion}

Follicular neoplasm carries a $15-30 \%$ risk of malignancy and typically requires thyroid lobectomy for diagnosis $[2,3]$. This suggests that up to $30 \%$ of patients will undergo reoperative completion thyroidectomy for treatment and cure. The utility of intraoperative FS in guiding surgical decision-making is debated. While our study was retrospective with a limited number of patients, it suggests that FS may be valuable in identifying malignancy early and thus reducing the need for reoperative surgery in patients with follicular neoplasm.

In this group of 148 subjects with follicular neoplasm on FNA, 51 (34\%) patients had a diagnosis of malignancy on final pathology. The likely explanation for the slightly higher than average rate of cancer in these patients was that our FNA samples were sent for second opinion pathologic review for a separate study. A third of patients had second opinion samples reported as benign compared to an initial diagnosis of follicular neoplasm, which may contribute to the higher number of cancer incidence [17]. While the overall risk of malignancy was $34 \%$ for follicular neoplasm, this number rose to $80 \%$ in 
patients with follicular neoplasm and suspicious FS findings. This suggests that FS aids the surgeon in identifying patients at risk of malignancy and thus can guide operative management when preoperative FNA is diagnostic of follicular neoplasm.

Our positive predictive value for FS in follicular neoplasms of the thyroid was $80 \%$, similar to quoted numbers in the literature (range 28.5-100\%) [5, 18-20]. Our negative predictive value of $77 \%$ is on the low end of those numbers quoted in the literature (range 74.1-95.3\%) [5, 18-20]. This may be due to our small sample size.

Thirteen of 67 (19\%) patients had a malignant final diagnosis despite unremarkable FS results. These patients therefore required reoperative completion thyroidectomy for cure. We suggest that if up to $30 \%$ of patients with follicular neoplasm have cancer, then FS may reduce the need for reoperative surgery and the risk of its associated complications by up to $11 \%$.

Of 79 patients who underwent intraoperative FS, five (6\%) had benign final pathology and thus unnecessarily endured total thyroidectomy and lifelong thyroid hormone replacement. Still, however, this number is quite small and many institutions are offering patients with follicular neoplasm total thyroidectomy from the onset; although, this remains a point of controversy. With this practice pattern, unnecessary total thyroidectomy may occur in $70-85 \%$ of patients with follicular neoplasm. On the other hand, a total of 20 patients warranted total thyroidectomy based on malignancy found on final pathology. Seven of these patients underwent total thyroidectomy at their initial operation based on findings which were suspicious on FS. Thus, we conclude that about $35 \%$ of patients avoided reoperative completion thyroidectomy based on FS results.

A total of 15 patients showed suspicious features on FS. Nine of these showed nuclear atypia, indicative of possible PTC, while six showed findings concerning for capsular or vascular invasion, a sign of FTC. Of the nine patients with nuclear atypia on FS, eight (89\%) had a final pathologic diagnosis of papillary carcinoma or papillary microcarcinoma. Of the six patients with features suspicious of FTC, three $(50 \%)$ had follicular carcinoma or Hurthle cell carcinoma on final pathology. We therefore suggest that intraoperative FS may be especially useful in recognizing nuclear abnormalities characteristic of papillary thyroid cancer.

In conclusion, this study demonstrates a number of possible benefits of intraoperative FS at the time of thyroid lobectomy for follicular neoplasm cytology diagnosis. A suspicious FS can increase the detection of malignancy when compared with preoperative FNA results alone. In our series, FS reduced the need for reoperative thyroid surgery in over a third of patients with malignant disease. We also found that patients with follicular neoplasm in a calcified thyroid nodule were very likely to have malignancy found on surgical pathology Finally, visualization of the features of PTC on FS are highly predictive of malignancy on surgical pathology.

\section{Acknowledgement}

The authors wish to thank Rutgers Robert Wood Johnson Medical School, Department of Surgery for its continued support in research endeavors.

\section{Financial Disclosures}

The participants in this study have no related to this research. Financial support was provided solely by the authors' contribution of personal time.

\section{Abbreviations}

FNA: fine needle aspiration; FS: frozen section; PTC: papillary thyroid carcinoma; FTC: follicular thyroid carcinoma

\section{References}

1. Cibas ES, Ali SZ. The Bethesda System For Reporting Thyroid Cytopathology. Am J Clin Pathol. 2009; 132(5):658-665.

2. Yang J, Schnadig V, Logrono R, Wasserman PG. Fineneedle aspiration of thyroid nodules: a study of 4703 patients with histologic and clinical correlations. Cancer. 2007;111(5):306-315.

3. Baloch ZW, Fleisher S, LiVolsi VA, Gupta PK. Diagnosis of "follicular neoplasm": a gray zone in thyroid fine-needle aspiration cytology. Diagn Cytopathol. 2002;26(1):4144.

4. Cooper DS, Doherty GM, Haugen BR, Kloos RT, Lee SL, Mandel SJ, Mazzaferri EL, et al. Revised American Thyroid Association management guidelines for patients with thyroid nodules and differentiated thyroid cancer. Thyroid. 2009;19(11):1167-1214.

5. Lumachi F, Borsato S, Tregnaghi A, Marino F, Polistina F, Basso SM, Koussis H, et al. FNA cytology and frozen section examination in patients with follicular lesions of the thyroid gland. Anticancer Res. 2009;29(12):52555257.

6. Osamura RY, Hunt JL. Current practices in performing frozen sections for thyroid and parathyroid pathology. Virchows Arch. 2008;453(5):433-440.

7. Cetin B, Aslan S, Hatiboglu C, Babacan B, Onder A, Celik A, Cetin A. Frozen section in thyroid surgery: is it a necessity? Can J Surg. 2004;47(1):29-33.

8. Hamming JF, Vriens MR, Goslings BM, Songun I, Fleuren GJ, van de Velde CJ. Role of fine-needle aspiration biopsy and frozen section examination in determining the extent of thyroidectomy. World J Surg. 1998;22(6):575579; discussion 579-580.

9. Chen H, Nicol TL, Udelsman R. Follicular lesions of the thyroid. Does frozen section evaluation alter operative management? Ann Surg. 1995;222(1):101-106.

10. Giuliani D, Willemsen P, Verhelst J, Kockx M, Vanderveken M. Frozen section in thyroid surgery. Acta Chir Belg. 2006;106(2):199-201.

11. Richards ML, Chisholm R, Bruder JM, Strodel WE. Is thyroid frozen section too much for too little? Am J Surg. 
2002;184(6):510-514; discussion 514.

12. Haymart MR, Greenblatt DY, Elson DF, Chen H. The role of intraoperative frozen section if suspicious for papillary thyroid cancer. Thyroid. 2008;18(4):419-423.

13. Makay O, Icoz G, Gurcu B, Ertan Y, Tuncyurek M, Akyildiz M, Yetkin E. The ongoing debate in thyroid surgery: should frozen section analysis be omitted? Endocr J. 2007;54(3):385-390.

14. Caraci P, Aversa S, Mussa A, Pancani G, Ondolo C, Conticello S. Role of fine-needle aspiration biopsy and frozen-section evaluation in the surgical management of thyroid nodules. Br J Surg. 2002;89(6):797-801.

15. Bugis SP, Young JE, Archibald SD, Chen VS. Diagnostic accuracy of fine-needle aspiration biopsy versus frozen section in solitary thyroid nodules. Am J Surg. 1986;152(4):411-416.

16. Chang HY, Lin JD, Chen JF, Huang BY, Hsueh C, Jeng LB, Tsai JS. Correlation of fine needle aspiration cytolo- gy and frozen section biopsies in the diagnosis of thyroid nodules. J Clin Pathol. 1997;50(12):1005-1009.

17. Davidov T, Trooskin SZ, Shanker BA, Yip D, Eng O, Crystal J, Hu J, et al. Routine second-opinion cytopathology review of thyroid fine needle aspiration biopsies reduces diagnostic thyroidectomy. Surgery. 2010;148(6):12941299; discussion 1299-1301.

18. Taneri F, Tekin E, Salman B, Anadol AZ, Ersoy E, Poyraz A, Onuk E. Huerthle cell neoplasms of the thyroid: predicting malignant potential. Endocr Regul. 2000;34(1):19-21.

19. Kahmke R, Lee WT, Puscas L, Scher RL, Shealy MJ, Burch WM, Esclamado RM. Utility of Intraoperative Frozen Sections during Thyroid Surgery. Int J Otolaryngol. 2013;2013:496138.

20. Liu FH, Liou MJ, Hsueh C, Chao TC, Lin JD. Thyroid follicular neoplasm: analysis by fine needle aspiration cytology, frozen section, and histopathology. Diagn Cytopathol. 2010;38(11):801-805. 